\section{Resolution of Cullen's sign in patient with metastatic melanoma responding to hypoxia-activated prodrug TH-302}

\author{
Glen J. Weiss, ${ }^{1}$ Karen Lewandowski, ${ }^{1}$ \\ Jon Oneall, ${ }^{1}$ Stew Kroll² \\ ${ }^{1}$ Virginia G. Piper Cancer Center Clinical \\ Trials Program at Scottsdale Healthcare, \\ Scottsdale, AZ; ${ }^{2}$ Threshold Pharmaceuticals, \\ Redwood City, CA, USA
}

\begin{abstract}
Cullen's sign, ecchymosis of the subcutaneous periumbilical tissue often described in association with non-malignant conditions such as ruptured ectopic pregnancy or acute pancreatitis, has been reported in malignancies involving the abdomen. In melanoma, hematoma-like metastasis has been observed and can resolve with an effective therapy. We observed resolution of Cullen's sign (probably hematoma-like metastasis) in a patient with metastatic melanoma. The patient was participating in a phase I clinical trial and treated with TH-302, a hypoxia-activated prodrug. After 2 months on study, complete resolution of Cullen's sign resolved in concert with extracranial response in lung, liver, and lymph node metastases. Based on the dramatic extracranial response to this investigational agent, additional patients with metastatic melanoma without evidence of brain metastasis were treated on study with TH-302.
\end{abstract}

\section{Introduction}

Cullen's sign, ecchymosis of the subcutaneous periumbilical tissue was often described in association with ruptured ectopic pregnancy or acute pancreatitis until 1922, when Stemnberg described its association with malignancy (adenocarcinoma of the liver). ${ }^{1}$ Since then, Cullen's sign associated with other malignancies involving the abdomen have been reported. In melanoma, hematoma-like metastasis $^{2-5}$ has been observed and can resolve with an effective therapy. ${ }^{5}$ Hematomalike metastases have not been reported to involve the periumbilical region, masquerading as Cullen's sign. This case report details the treatment course of a patient with metastatic melanoma with resolution of Cullen's sign (probably hematoma-like metastasis) with tumor response to TH-302, a hypoxia-activated prodrug, in a phase I clinical trial, ${ }^{6}$ leading to further investigation of $\mathrm{TH}-302$ in patients with metastatic melanoma.?

\section{Case Report}

A 74-year-old Caucasian man presented to our clinic with metastatic melanoma (MM) involving the lung and liver. Seventeen months prior, he was diagnosed with superficial spreading melanoma on his upper back, Clark level IV, Breslow thickness $1.71 \mathrm{~mm}$ with four mitotic figures per high power field. No lymphangitic or perineural invasion was identified. He underwent wide excision with margins of $1 \mathrm{~cm}$. Subsequently, on surveillance CT scans, lung and liver masses suspicious for metastases were identified. CT-guided biopsy of a lung mass confirmed metastatic disease and DTIC was initiated. Initial scans showed a favorable PET/CT response. Three months later, the patient complained of epigastric discomfort and pain and CT scans, a few weeks before our evaluation, demonstrated disease progression.

Other past medical history was unremarkable except for remote history of early-stage prostate cancer resected approximately eight years prior, medication controlled hypertension, and hearing aid use. His only symptoms were pain with deep inspiration and intermit tent pain in the right flank. His Karnofsky Performance Status was 90.

Physical examination was significant for healed surgical scar on the upper back from melanoma removal, firm abdomen and liver palpated $4 \mathrm{~cm}$ below the costophrenic margin without splenomegaly, and asymptomatic periumbilical ecchymosis, consistent with Cullen's sign (Figure 1A). Neurological exam was only significant for bilateral hearing loss. Laboratory evaluation revealed an elevated lactate dehydrogenase (LDH) of 1,004 IU/L (ULN $=500 \mathrm{IU} / \mathrm{L}$ ) and absence of coagulation abnormalities. After signed informed consent, patient was screened and enrolled on the TH302 phase I trial ${ }^{6}$ at a dose of $670 \mathrm{mg} / \mathrm{m}^{2}$ i.v. weekly x 3 every 28 days. 0 C CT imaging prior to dosing, a $3.8 \mathrm{~cm}$ lobulated, left lower lung mass (Figure 2A), $2.7 \mathrm{~cm}$ paracaval lymph node, and multiple liver masses (largest was $13.3 \mathrm{~cm}$ ) were identified (Figure 2C). Prior to his second weekly dose, asymptomatic blistering and scabbing of skin on the medial surface of the right arm near the i.v. infusion site was observed. He noted a decrease in epigastric discomfort. The second and third doses were delivered in the opposite arm. In his off-dosing week, he reported to his primary oncologist with bilateral upper extremity swelling and was diagnosed with superficial thrombophlebitis after Doppler ultrasound. No pul-
Correspondence: Glen J. Weiss, Virginia G. Piper Cancer Center Clinical Trials Program at Scottsdale Healthcare, 10510 N. 92nd St., Ste 200, Scottsdale, AZ 85238, USA.

Tel. +1.480.323.1350 - Fax: +1.480.323.1359.

E-mail:gweiss@tgen.org

Key words: Cullen's sign, TH-302, melanoma, hypoxia-activated prodrug.

Acknowledgment: we are indebted to the patient and his family, providers, and staff and Dr. John Curd for their contributions.

Contributions: GJW, study conception and design, data acquisition and interpretation, manuscript draft and revised; KL, J0, data acquisition, administrative and technical support; SK, study conception and design, data acquisition and interpretation, manuscript draft and revised.

Disclosure: Stew Kroll is employed by Threshold Pharmaceuticals and owns stock in the company. The other authors have no disclosures.

Funding: this study was supported in part by Threshold Pharmaceuticals.

Received for publication: 27 0ctober 2011. Accepted for publication: 31 October 2011.

This work is licensed under a Creative Commons Attribution NonCommercial 3.0 License (CC BYNC 3.0).

(C) Copyright G.J. Weiss et al., 2011

Licensee PAGEPress, Italy

Dermatology Reports 2011; 3:e56

doi:10.4081/dr.2011.e56

monary embolism was identified on CT angiography. He was started on low molecular weight heparin (LMWH) and coumadin. At cycle 2 week 1 dosing, mild-moderate fatigue, dry non-productive cough, and petechial rash on his lower extremities were observed. Physical exam revealed a softer abdomen and significantly smaller liver, now palpable to $2 \mathrm{~cm}$ below the costophrenic margin, with less prominent periumbilical ecchymosis. He also had mild petechial rash of the lower extremities and perianal redness, causing minimal discomfort. Bilateral extremities had blistering and healing scabs. No new neurological findings were identified.

Evaluation of his outside CT angiogram revealed improvements in target lesion diameters by RECIST criteria (22\% decrease). ${ }^{8}$ After insertion of a central line, cycle 2 was initiated with heat packs and NSAIDs for superficial thrombophlebitis per guidelines. ${ }^{9}$

On cycle 2 week 2 visit, patient reported increased pain, swelling, and palpable cord in his right arm for three days. He also noted an ulceration on his left arm near the site of prior i.v. insertion, as well as, mild rectal pain and 
bright red blood after wiping. Physical exam revealed continued decrease in palpable liver and near resolution of periumbilical ecchymosis. A palpable firm and tender cord was noted in the right forearm, and rectal examination revealed no ulceration, hemorrhoid, mass, or gross blood. Doppler ultrasound of the upper
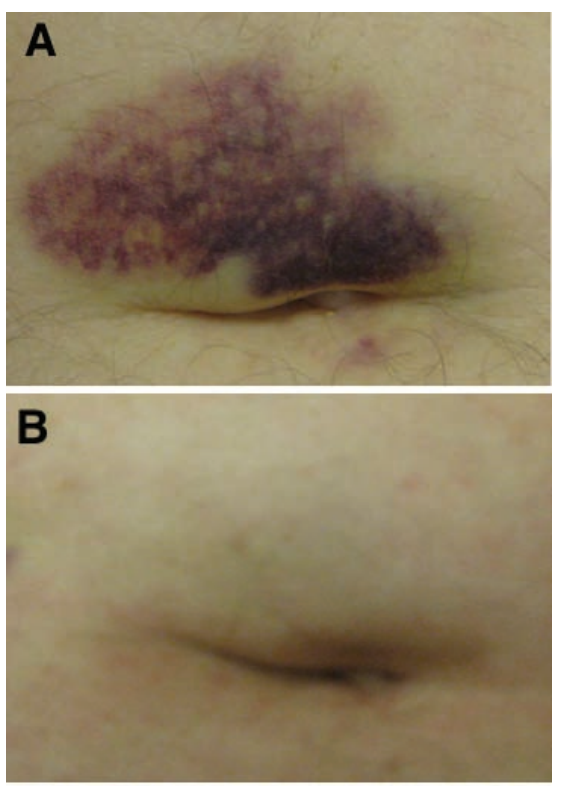

Figure 1. A)Photograph of Cullen's sign at baseline. B) Photograph of Cullen's sign prior to cycle 3 .
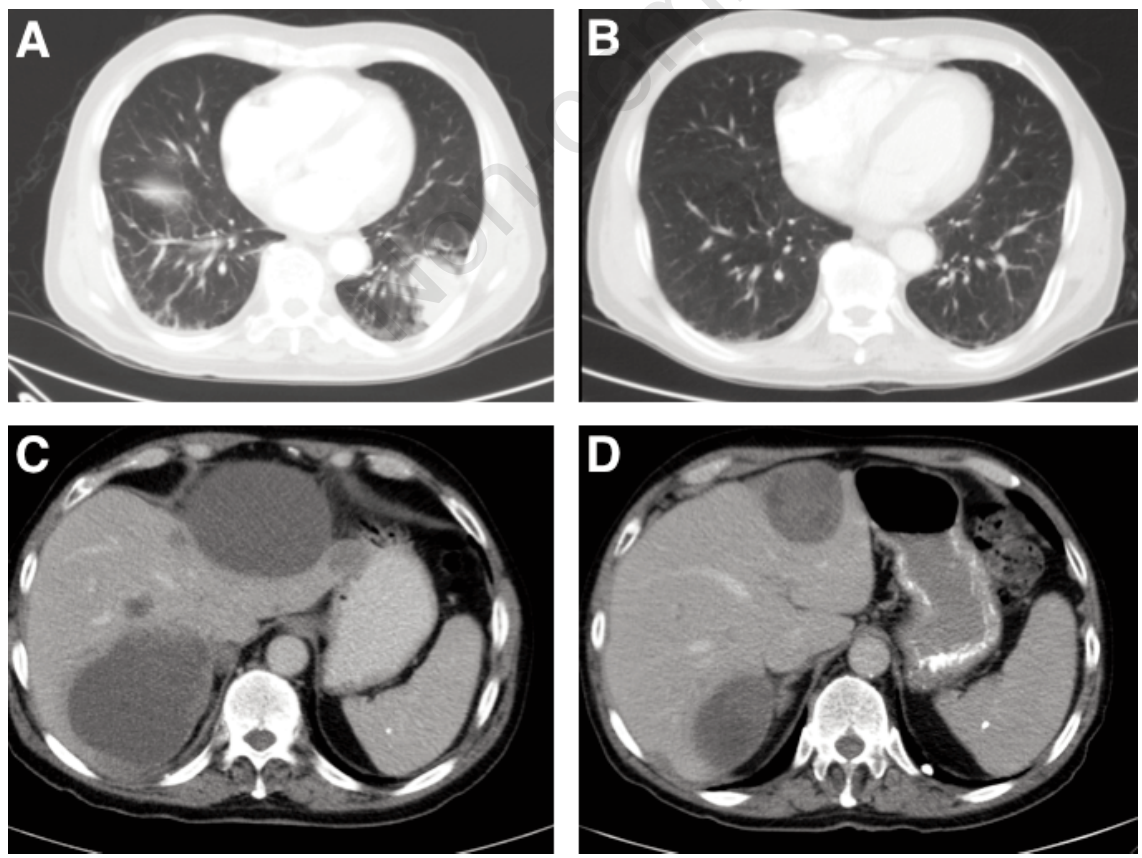

Figure 2 A) Computed tomography scan image depicting lung mass at baseline. B) Computed tomography scan image depicting lung mass prior to cycle 3 . C) Computed tomography scan image depicting representative liver metastases at baseline. D) Computed tomography scan image depicting representative liver metastases prior to cycle 3. until the time the seizures began, there was no new neurological finding prior to start of the study or during the treatment. It is probable that brain metastasis were present but unrealized at the initiation of treatment and then progressed, and less likely that new brain metastasis developed while the patient's extracranial sites were significantly decreasing in size. Based on the dramatic extracranial response, additional patients with MM have been treated with TH-302 at the slightly lower MTD dose of $575 \mathrm{mg} / \mathrm{m}^{2}$.

\section{References}

1. Mabin TA, Gelfand M. Cullen's sign, a feature in liver disease. BMJ 1974;1:493-4.

2. Torok L, Kirschner A, Ócsai H, Olasz K. Hematoma-like metastasis in melanoma. J

Am Acad Dermatol 2003;49:912-3.

3. Jouary T, Delaunay M, Taieb A. Case letters: hematoma-like metastases. J Am Acad Dermatol 2006;55:1106-7.

4. Connolly CM, Soldin M, Cooper AC, Dawson A. Metastatic malignant melanoma presenting with a bruise. Br J Plast Surg 2003;56:76.

5. Dehart WK, Gilliam AC, Lu KQ, Brell J. A rare case of melanoma recurring as subcutaneous metastatic melanoma with overlying ecchymoses. Arch Dermatol 2008;144: 561-2.

6. Weiss GJ, Infante JR, Chiorean EG, et al Phase 1 Study of the Safety, Tolerability and Pharmacokinetics of TH-302, a Hypoxia-Activated Prodrug, in Patients with Advanced Solid Malignancies. Clin Cancer Res 2011 Mar 17. [Epub ahead of print]

7. Curd JG, Infante R, Weber R, et al. Initial Clinical Observations Regarding TH-302, a Tumor Selective Hypoxia-Activated Prodrug, in Metastic Melanoma. Melanoma Perspective XIII meeting, 2009. Available from: http://www.thresholdpharm.com/sec/posters

8. Therasse P, Arbuck SG, Eisenhauer EA, et al. New guidelines to evaluate the response to treatment in solid tumors. European Organization for Research and Treatment of Cancer, National Cancer Institute of the United States, National Cancer Institute of Canada. J Natl Cancer Inst 2000;92:205-16.

9. Kearon C, Kahn SR, Agnelli G, et al Antithrombotic therapy for venous thromboembolic disease: American College of Chest Physicians Evidence-Based Clinical Practice Guidelines (8th Edition). Chest 2008;133:454S-545S. 